\title{
Estimation of BRDF model kernel weights under an a priori knowledge-aided constraint
}

\section{Xinpeng Tian, Zhiqiang Gao, Qiang Liu, Yueqi Wang \& Xiuhong Li}

To cite this article: Xinpeng Tian, Zhiqiang Gao, Qiang Liu, Yueqi Wang \& Xiuhong Li (2021)

Estimation of BRDF model kernel weights under an a priori knowledge-aided constraint, Remote Sensing Letters, 12:2, 150-159, DOI: 10.1080/2150704X.2020.1823036

To link to this article: https://doi.org/10.1080/2150704X.2020.1823036

曲 Published online: 20 Jan 2021.

Submit your article to this journal

Џ Article views: 45

Q View related articles $\sqsubset$

View Crossmark data $₫$ 


\title{
Estimation of BRDF model kernel weights under an a priori knowledge-aided constraint
}

\author{
Xinpeng Tian $\mathbb{D}^{\mathrm{a}, \mathrm{b}}$, Zhiqiang Gao ${ }^{\mathrm{a}, \mathrm{b}}$, Qiang Liu ${ }^{\mathrm{c}, \mathrm{d}}$, Yueqi Wang ${ }^{\mathrm{a}, \mathrm{b}}$ and Xiuhong Li $\mathrm{Li}^{\mathrm{c}, \mathrm{d}}$
}

${ }^{a}$ CAS Key Laboratory of Coastal Environmental Processes and Ecological Remediation, Yantai Institute of

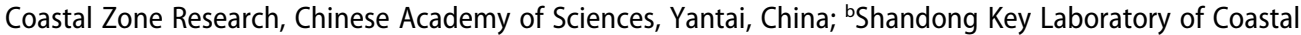
Environmental Processes, Yantai Institute of Coastal Zone Research, Chinese Academy of Sciences, Yantai, China; ' ${ }^{C}$ ollege of Global Change and Earth System Science, Beijing Normal University, Beijing, China; ${ }^{d}$ State Key Laboratory of Remote Sensing Science, Jointly Sponsored by Beijing Normal University and the Institute of Remote Sensing and Digital Earth of Chinese Academy of Sciences, Beijing, China

\section{ABSTRACT}

The reflectance anisotropy of land surface serves as an important bridge between surface biophysical parameters and remote sensing observations. It can characterize by the linear kernel-driven bidirectional reflectance distribution function (BRDF), which is the combination of several kernel functions and kernel weights. These kernel weights can be estimated by remote sensing; however, the stability of current kernel weights products is still challenging, especially in urban areas with complex aerosol properties and heterogeneous surfaces. In this paper, we propose a method for robust estimation of kernel weights from the Moderate Resolution Imaging Spectroradiometer (MODIS) surface spectral reflectance products (MxD09GA) data based on the constrained least-squares method (CLSM) and a priori knowledge. The kernel weights data were obtained by the CLSM from 2014 to 2017 in Beijing region of China. Validations were carried out using the MxD09GA and BRDF/Albedo products (MCD43A1). The results show that the time series of kernel weights by the CLSM show small variability over different land cover types. The kernel weights estimated by the CLSM can clearly show the phenological signal and fitting ability of surface spectral reflectance is better than that of the MCD43A1 products in Beijing urban area. Experimental results demonstrate that the CLSM has the potential for the robust estimation of kernel weights in urban areas.

\section{ARTICLE HISTORY}

Received 25 April 2020

Accepted 8 September 2020

\section{Introduction}

Anisotropy is a fundamental physical property of land surface reflectance, and it plays an important role in acquiring quantitative information about the earth's surface from data obtained by remote sensing instrument. The anisotropic scattering behaviour of land surface is described by the bidirectional reflectance distribution function (BRDF), which is a mathematical function of viewing and illumination geometries (Nicodemus et al. 1977). The BRDF is one of the important factors to be considered when utilizing satellite data with a large range of view or sun zenith angles, such as Moderate Resolution Imaging Spectroradiometer (MODIS). The influence of geometric angles cannot be ignored when the MODIS data are used to retrieve land or atmosphere products. The impact of 
surface anisotropy on sensor observations has been a focus in remote sensing application researches (Trigg, Roy, and Flasse 2005; Zhang et al. 2018).

The kernel-driven BRDF models are regarded as robust semiempirical BRDF models, which can be applied to most land cover types (Susaki et al. 2004) and widely used to describe BRDF properties (Tang et al. 2007). These models can reduce the difficulties of physical BRDF models by approximating the original physical process. Kernel-driven BRDF models are generally composed of several kernel functions and kernel weights. The expression of kernel function is related to the land cover types (Gao et al. 2000; Wanner, Li, and Strahler 1995). The estimation of kernel weights is affected by various factors, including errors of the atmospheric correction, surface reflectance data with insufficient angular range of measurements and model-fitting method without resistant to noise (Knobelspiesse et al. 2008).

The robustness of kernel weights estimation is an important prerequisite when correcting remotely sensed data in order to obtain meaningful physical parameters. In general, the estimation of kernel weights is solved by minimizing the sum of the squared fitting error (Susaki et al. 2004). However, the errors and poor angle ranges of surface reflectance data reduce the accuracy of kernel weights estimation or even fail. Some studies show that a priori knowledge can significantly improve the retrieval of albedo and bidirectional reflectance (Li et al. 2001). For those cases with insufficient or poor sampling, or a poor fit, a magnitude inversion is performed rather than a full model inversion. The early efforts by Strugnell and Lucht used 68 field multi-angle measurements to derive 25 socalled BRDF-based classes by cross walking with ecological land cover types. There is anisotropic scattering information on more complex heterogeneous environments. In order to captures this anisotropic scattering information, a new vegetation index, the Anisotropic Flat Index (AFX), is proposed (Jiao et al. 2014).

Currently, various kernel-driven BRDF products with different spatial and temporal resolutions have been derived from different satellite platforms (Wang et al. 2018). These products significantly advance the progress of quantitative land surface remote sensing. However, the retrieved kernel weights from remote sensing observations is still a challenge, especially in urban areas with bright and heterogeneous surface. The surface reflectance data are often contaminated by errors caused by imperfect cloud mask and atmospheric correction (Vermote, El Saleous, and Justice 2002b), the errors will be brought to the estimation of BRDF model parameters (Lucht and Roujean 2000). In order to reduce the uncertainty of surface reflectance data, a robust estimation of BRDF model parameters is necessary. In this paper, the constrained least-squares method (CLSM) and a priori knowledge are used to estimate kernel weights and ensure the robustness of inversion results. We focused on the CLSM as a robust kernel weights estimator and tested it in Beijing region using multi-year MODIS daily surface spectral reflectance product (MxD09GA, where $\mathrm{x}$ is ' $\mathrm{O}$ ' for Terra and ' $\mathrm{Y}$ ' for Aqua). The CLSM retrievals are compared with MODIS BRDF/Albedo model parameters product (MCD43A1), and the results show that the robustness of the CLSM retrievals is better than that of the MCD43A1 products in Beijing urban area.

\section{Study area and dataset}

\subsection{Study area}

The study area of Beijing is located in the north-western edge of the North China Plain, with latitude from $39^{\circ} 28^{\prime} \mathrm{N}$ to $41^{\circ} 05^{\prime} \mathrm{N}$ and longitude from $115^{\circ} 25^{\prime} \mathrm{E}$ to $117^{\circ} 30^{\prime} \mathrm{E}$, as shown in Figure 1. The land cover types of Beijing are mainly composed of three types, 
including natural vegetation (forest, grassland, and shrubland), cultivated land, and artificial surfaces. Some studies show that the directional reflection characteristics over urban and vegetation areas are more evident than that over other areas, such as desert and semidesert (Noguchi et al. 2014). To evaluate the applicability and stability of the CLSM for kernel weights retrievals, in this study, a total of 37 sites were selected for validation, including 14 natural vegetation sites, 11 artificial surface sites and 12 cultivated land sites. The spatial distribution of all sites is shown in Figure 1.

\subsection{MODIS products}

The MxD09GA product provides the daily surface spectral reflectance for each of the MODIS bands 1 through 7 with a $500 \mathrm{~m}$ spatial resolution. The Collection 6 (C6) MCD43A1 product provides the daily isotropic, volumetric and geometric kernel weights, while the MODIS vegetation indices product (MOD13A1) provides two vegetation indexes, including enhanced vegetation index and normalized difference vegetation index (NDVI). In this study, the 4-year (2014-2017) MxD09GA data in two tiles (H26V04, H26V05) were filtered using their internal quality assurance flags (QA) to provide surface spectral reflectance data, the MCD43A1 and MOD13A1 data were utilized to acquire a priori knowledge of kernel weights under different vegetation states, and only highest-quality ( $Q A=0$ for the MCD43A1 data) retrievals are selected. Besides, the MCD43A1 and MxD09GA products are also used to verify the accuracy of the CLSM retrievals over different land cover types.

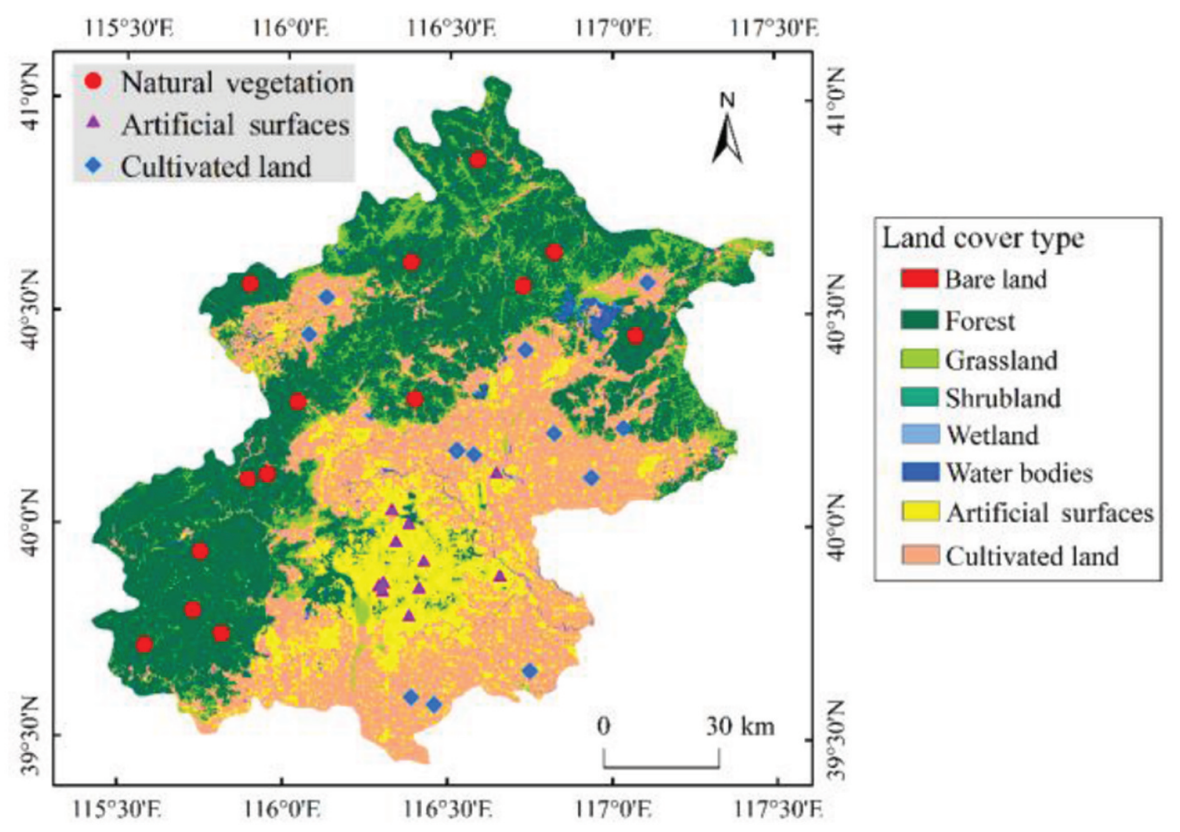

Figure 1. Location of 37 sites in Beijing area. Background map is land cover-type dataset, which is provided by GlobeLand30. 


\section{Methodology}

The linear kernel-driven BRDF model typically consists of three scattering components, including isotropic, volumetric and geometric scattering, it can be expressed as (Lucht, Schaaf, and Strahler 2000):

$$
\rho\left(\theta_{\mathrm{s}}, \theta_{\mathrm{v}}, \varphi, \lambda\right)=f_{\text {iso }}(\lambda)+f_{\mathrm{vol}}(\lambda) K_{\mathrm{vol}}\left(\theta_{\mathrm{s}}, \theta_{\mathrm{v}}, \varphi\right)+f_{\mathrm{geo}}(\lambda) K_{\mathrm{geo}}\left(\theta_{\mathrm{s}}, \theta_{\mathrm{v}}, \varphi\right)
$$

where $\rho$ is the surface bidirectional reflectance, $\lambda$ is the wavelength, $K_{\text {vol }}$ and $K_{\text {geo }}$ are kernel functions of solar zenith angle $\theta_{s}$, view zenith angle $\theta_{v}$ and relative azimuth angle $\varphi$, describe the surface volumetric and geometric-optical scattering, respectively. $f_{\text {vol }}$ and $f_{\text {geo }}$ represent the weight coefficients for both kernels, $f_{\text {iso }}$ is a constant corresponding to isotropic reflectance. The combination of the RossThick-LiSparseR kernel functions (RTLSR) has a good agreement with observation data (Lucht and Lewis 2000). The 'RossThick' kernel (Ross 1981), applies a single-scattering radiative transfer model for a uniform dense leaf canopy with a Lambertian background. The 'LiSparse-R' kernel (Li and Strahler 1992), considers the shadow-casting effect on reflectance, based on the height and shape of objects, for a sparse canopy with a Lambertian background. This model has been greatly developed in some aspects, including its hotspot effects (Jiao et al. 2016) and its ability in modelling snow reflectance (Ding et al. 2019). The RTLSR kernel-driven BRDF model is used in the MODIS at-launch AMBRALS (Algorithm for MODIS Bidirectional Reflectance Anisotropies of the Land Surface).

For a vector $\rho$, Equation (1) can be expressed in matrix form:

$$
\rho\left(\theta_{\mathrm{s}}, \theta_{\mathrm{v}}, \varphi, \lambda\right)=\mathbf{K}\left(\theta_{\mathrm{s}}, \theta_{\mathrm{v}}, \varphi\right) f(\lambda)
$$

where $f$ and $\mathbf{K}$ are the matrices of kernel weights and kernel functions, respectively. These matrices are given by:

$$
\begin{gathered}
\rho=\left[\begin{array}{llll}
\rho_{1} & \rho_{2} & \cdots & \rho_{N}
\end{array}\right]^{\top} \\
f=\left[\begin{array}{lll}
f_{\text {iso }} & f_{\text {vol }} & f_{\text {geo }}
\end{array}\right]^{\top} \\
\mathbf{K}=\left[\begin{array}{ccc}
1 & K_{1}^{\text {vol }} & K_{1}^{\text {geo }} \\
1 & K_{2}^{\text {vol }} & K_{2}^{\text {geo }} \\
\cdots & \cdots & \cdots \\
1 & K_{N}^{\text {vol }} & K_{N}^{\text {geo }}
\end{array}\right]
\end{gathered}
$$

where $N$ is the number of directional reflectance data.

When $N$ is greater than three, $f$ can be solved by the least-squares method (LSM) of the overdetermined equation, and its cost function $J(f)$ is as follows:

$$
J(f)=(\mathbf{K} f-\rho)^{\top}(\mathbf{K} f-\rho)
$$

The standard LSM for $f$ is obtained by:

$$
f=\left(\mathbf{K}^{\top} \mathbf{K}\right)^{-1} \mathbf{K}^{\top} \rho
$$

In the process of solving, Equation (7) must be applied across some time window so that sufficient samples are collected to solve for $f$ and compensate for error in the $\rho$ data. In the MCD43A1 product using spectral reflectance data from both Aqua and Terra satellites, the time window is set to 8 days $(N=16)$. 
The standard LSM can normally provide good fitting results. However, the implementation of the least-squares criterion lacks robustness when there are the large errors or noises in the reflectance data $\rho$. Numerical experiments show that if the LSM is used to calculate $f$, the results are very sensitive to the noises (Quaife and Lewis 2010). Thus, we proposed to adopt CLSM to estimate $f$, in order to ensure the robustness of the estimation results. The CLSM can obtain a reasonable solution by adding a priori knowledge into the regression model. The cost function of the CLSM with the a priori knowledge-assisted constraints can be expressed as:

$$
J(f)=(\mathbf{K} f-\rho)^{\top} \boldsymbol{\Sigma}^{\top}(\mathbf{K} f-\rho)+\left(f-f_{0}\right)^{\top} \mathbf{M}^{\top}\left(f-f_{0}\right)=J_{\mathrm{x}}+J_{\mathrm{y}}
$$

where $\boldsymbol{\Sigma}$ is a covariance matrix of the surface reflectance, $f_{0}$ is the a vector of kernel weights average values and $\mathbf{M}$ is a covariance matrix of kernel weights, respectively. $J_{x}$ indicates the fitting results of the simulated and inputted surface reflectance data, while $J_{y}$ indicates the coincidence degree of the estimated and prior kernel weights.

The least-squares solution of a priori knowledge-aided constraint is given by:

$$
f=\left(\mathbf{K}^{\top} \boldsymbol{\Sigma}^{-1} \mathbf{K}+\mathbf{M}^{-1}\right)^{-1}\left(\mathbf{K}^{\top} \boldsymbol{\Sigma}^{-1} \rho+\mathbf{M}^{-1} f_{0}\right)
$$

Because the uncertainty of the MxD09GA product is small in the blue band (Vermote, El Saleous, and Justice 2002a), the diagonal matrix $\boldsymbol{\Sigma}$ can be set to 0.02 based on simple statistics of the MxD09GA products in this study. At present, the vegetation status of the four seasons is divided into two categories according to the NDVI in the study area. Then, a priori knowledge of the average values $f_{0}$ and covariance matrix $\mathbf{M}$ in Equation (9) were obtained from the MCD43A1 products, and the statistical results are shown in Table 1. Here, it should be noted that even if the constraints are not very precise, the negative effect on kernel weights estimation will not be significant, but the benefit to the stability will be very obvious.

\section{Results and discussion}

\subsection{Spatial coverage analysis}

Figure 2 presents the spatial distributions of kernel weights $\left(f_{\text {iso, }}, f_{\text {vol }}\right.$ and $\left.f_{\text {geo }}\right)$ from the CLSM retrievals and MCD43A1 products on 24 February 2014 in the study area. The $f_{\text {iso }}$ of the CLSM retrievals shows a similar spatial trends to that of the MCD43A1 products, with low values in the forest over the mountainous areas, and high values in agricultural and built-up areas on the flat plains. The absolute difference of the $f_{\text {iso }}$ between the CLSM retrievals and MCD43A1 products is approximately 0.01 . Compared with the MCD43A1 products, the $f_{\text {vol }}$ and $f_{\text {geo }}$ of the CLSM retrievals show more dependence on land cover types. In addition, the spatial coverage of all kernel weights generated by the CLSM has been extended from the forest and agricultural areas to the built-up areas, with almost no gaps except for the water areas. It should be noted that the $f_{\text {iso }}$ is higher than $f_{\text {vol }}$ and $f_{\text {geo }}$ in the study area, which means that the contribution of isotropic reflectance is the largest when the kernel-driven BRDF model is used to calculate the surface reflectance.

\subsection{Time-series analysis}

To further investigate the trends in the kernel weights, we examine data from both of the CLSM retrievals and MCD43A1 products. These data are monthly average of kernel weights over different land cover types from 2014 to 2017. Figure 3 shows that the three kernel weights 
from the CLSM retrievals and MCD43A1 products have similar trends over different land cover types. We can see that the artificial surfaces type, which is a major land cover type, has small seasonal dependence. This is consistent with the fact that urban surface features (e.g., buildings and roads) are basically permanent, although plants beside roads and in parks possibly bring about some seasonality (Noguchi et al. 2014). The natural vegetation in the study area is deciduous forest with contrastive seasonality, the kernel weights of the deciduous forest type largely depend on season. The difference is caused by winter leaf drop in deciduous forests. The phenological signal is clear with leaf-out occurring between April and October marked in the $f_{\text {iso }}$ by a decline in the blue band. This fits the expectation of photon absorption in the canopy, where blue light will be strongly absorbed by an abundance of green leaves. Unlike $f_{\text {iso, }}$ as shown in Figure $3(\mathrm{~b})$, the $f_{\text {vol }}$ has a small variation over all land cover types in the whole year, and the average value of all results is about 0.035 . There is no clear phenological signal in the $f_{\text {vol }}$ for either the CLSM retrievals or MCD43 products.

\subsection{Predicting surface spectral reflectance}

To evaluate the performance of the CLSM, it is necessary to test the fitting of kernel weights to surface spectral reflectance. In this paper, the observed surface spectral reflectance data are obtained from the Visible Infrared Imaging Radiometer Suite (VIIRS) daily surface reflectance Version 1 product (VNP09GA) (hereinafter called as the VNP09_SR), and the predicted surface spectral reflectance data are calculated by Equation (1) using kernel weights from the CLSM retrievals and MCD43A1 products (hereinafter called as the CLSM_SR and MCD43_SR, respectively). Then, the daily average spectral reflectance is calculated from 2014 to 2017 for these surface spectral reflectance. Figure 4(a,b) show the scatter plots of the CLSM_SR and MCD43_SR, respectively, with the VNP09_SR. The statistical indicators, including the correlation coefficient $(r)$, mean absolute error (MAE), and root mean square error (RMSE), are calculated over different land cover types (Table 2). As shown in Figure 4 and Table 2, the results of the CLSM_SR appear to lie evenly around the 1:1 line and exhibit only a small bias. The CLSM_SR agree well with VNP09_SR, with a high $r$ of 0.938 and low MAE and RMSE errors of approximately 0.009 and 0.014 , respectively. A higher $r$ of 0.868 and 0.938 with lower MAE and RMSE errors were observed from the CLSM retrievals than from the MCD43A1 products where the $r$ is 0.812 and 0.911 over artificial surface and cultivated land types, respectively.

Table 1. A priori knowledge of kernel weights average value and covariance matrix from the MCD43A1 products. $f_{\mathrm{i}-\mathrm{j}}$ is the covariance value between $\mathrm{i}$ and $\mathrm{j}, \mathrm{i}, \mathrm{j}=\mathrm{iso}$, vol and geo.

\begin{tabular}{|c|c|c|c|c|c|c|c|c|c|}
\hline \multirow[b]{2}{*}{$\begin{array}{l}\text { A priori } \\
\text { knowledge }\end{array}$} & & \multicolumn{2}{|c|}{ Spring } & \multicolumn{2}{|c|}{ Summer } & \multicolumn{2}{|c|}{ Autumn } & \multicolumn{2}{|c|}{ Winter } \\
\hline & & $\begin{array}{c}\mathrm{NDVI}< \\
0.4\end{array}$ & $\begin{array}{c}\text { NDVI > } \\
0.4\end{array}$ & $\begin{array}{c}\text { NDVI < } \\
0.4\end{array}$ & $\begin{array}{c}\text { NDVI > } \\
0.4\end{array}$ & $\begin{array}{c}\text { NDVI }< \\
0.4\end{array}$ & $\begin{array}{c}\mathrm{NDVI}> \\
0.4\end{array}$ & $\begin{array}{c}\mathrm{NDVI}< \\
0.2\end{array}$ & $\begin{array}{c}\mathrm{NDVI}> \\
0.2\end{array}$ \\
\hline Average & $f_{\text {iso }}$ & 0.1425 & 0.0917 & 0.0912 & 0.0395 & 0.0965 & 0.0674 & 0.1526 & 0.1193 \\
\hline value & $f_{\mathrm{vol}}$ & 0.0651 & 0.0333 & 0.0303 & 0.0288 & 0.0284 & 0.0266 & 0.0577 & 0.0399 \\
\hline$\left(f_{0}\right)$ & $f_{\text {geo }}$ & 0.0248 & 0.0159 & 0.0207 & 0.0285 & 0.0238 & 0.0178 & 0.0319 & 0.0289 \\
\hline Covariance & $f_{\text {iso-iso }}$ & 0.0009 & 0.0008 & 0.0006 & 0.0002 & 0.0007 & 0.0003 & 0.0010 & 0.0015 \\
\hline \multirow[t]{6}{*}{ (M) } & $f_{\text {iso-vol }}$ & -0.0004 & -0.0002 & 0.0001 & 0.0000 & 0.0000 & 0.0000 & -0.0001 & 0.0001 \\
\hline & $f_{\text {iso-geo }}$ & 0.0002 & 0.0002 & 0.0002 & 0.0001 & 0.0002 & 0.0001 & 0.0004 & 0.0004 \\
\hline & $f_{\mathrm{vol}-\mathrm{vol}}$ & 0.0011 & 0.0008 & 0.0005 & 0.0003 & 0.0004 & 0.0004 & 0.0013 & 0.0008 \\
\hline & $f_{\text {vol-geo }}$ & -0.0002 & -0.0001 & -0.0001 & 0.0000 & -0.0001 & -0.0001 & 0.0001 & 0.0000 \\
\hline & $f_{\text {geo- }}$ & 0.0001 & 0.0001 & 0.0001 & 0.0000 & 0.0001 & 0.0001 & 0.0002 & 0.0002 \\
\hline & geo & & & & & & & & \\
\hline
\end{tabular}




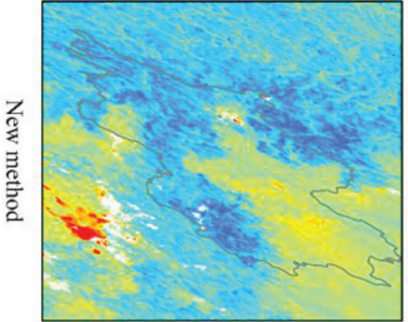

(a)

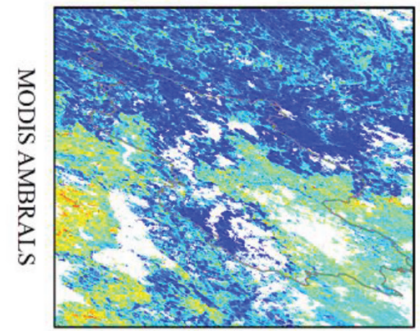

(d) 0.05

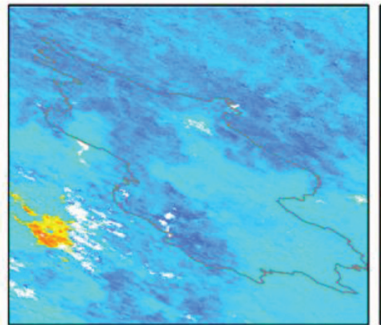

(b)

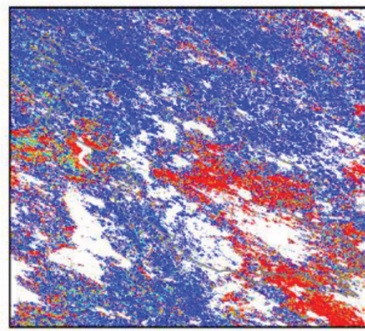

(e)

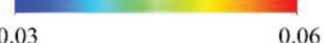

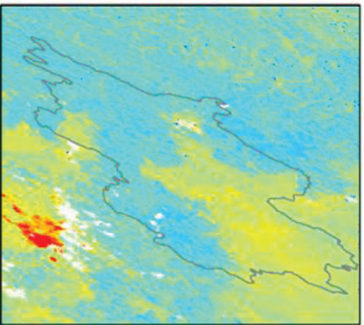

(c)

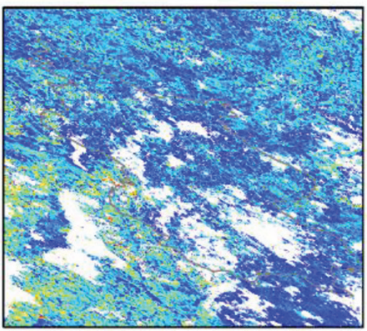

(f)

0.0

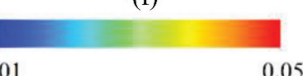

Figure 2. Spatial patterns of kernel weights obtained from the CLSM retrievals and MCD43A1 products (only pixels marked as best quality) over Beijing region on 24 February 2014. (a, d) isotropic kernel weight, (b, e) volumetric kernel weight, (c, f) geometric kernel weight.
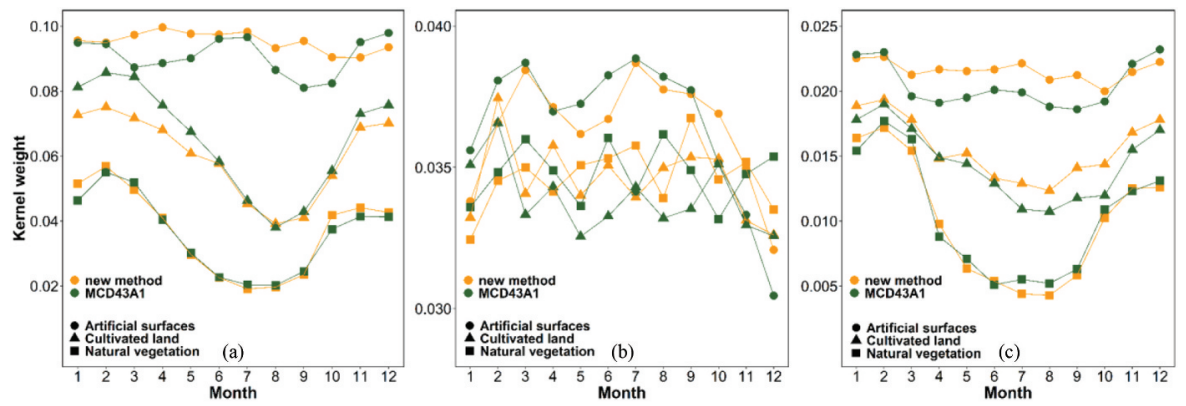

Figure 3. Temporal trajectories of kernel weights obtained from the CLSM and MCD43A1 products over different land cover types. (a) isotropic kernel weight, (b) volumetric kernel weight, and (c) geometric kernel weight.

\section{Conclusion}

To obtain stable estimation results of kernel weights from MODIS data over Beijing urban area, in this letter, the constrained least-squares method (CLSM) was investigated as robust parameter estimators, and the AMBRALS method was compared to the estimation results. The major difference between the AMBRALS and CLSM method is that CLSM uses a priori knowledge to impose expectations on the resulting kernel weights. The approach using the CLSM to constrain the kernel weights retrievals works well over most land cover types. The phenological signals are well described in the kernel weights retrieved by the CLSM over vegetation areas. The 

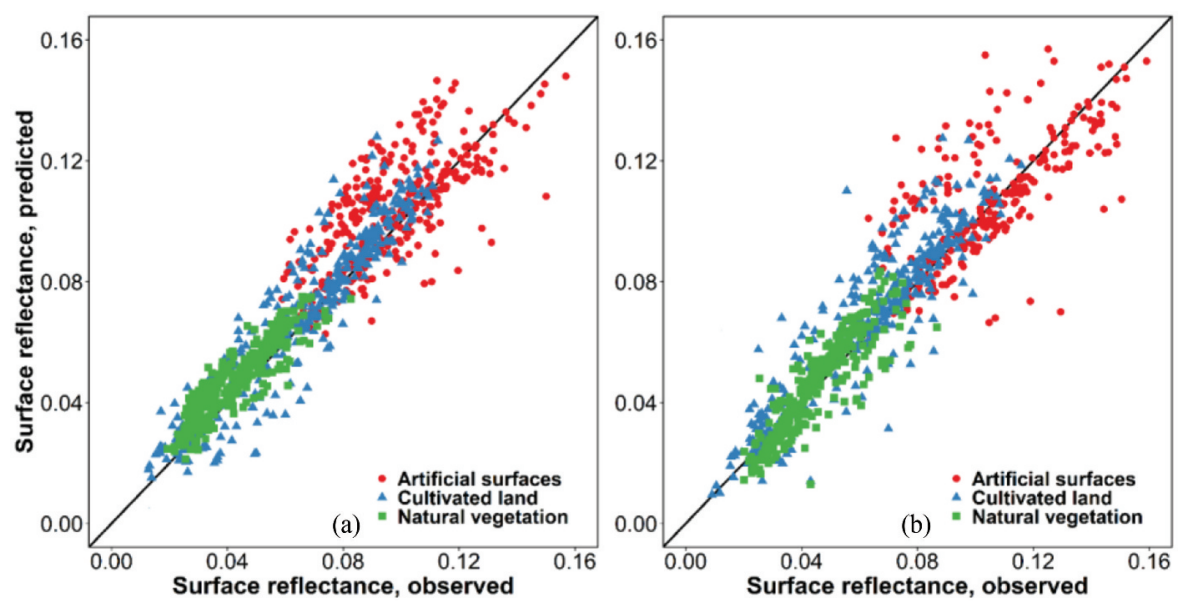

Figure 4. Scatter plots of predicted surface spectral reflectance data are calculated by kernel-driven BRDF model using kernel weights derived with the (a) CLSM retrievals and (b) MCD43A1 products. The observed surface spectral reflectance data are obtained from VNP09GA products.

Table 2. Descriptive statistics of the predicted surface reflectance data from kernel-driven BRDF model using kernel weights derived with the CLSM retrievals and MCD43A1 products. $r$ is the correlation coefficient between predicted and observed surface reflectance data, MAE is the mean absolute error and RMSE is the root mean square error.

\begin{tabular}{lcccccccccccc}
\hline & \multicolumn{3}{c}{ Natural vegetation } & \multicolumn{4}{c}{ Artificial surface } & \multicolumn{3}{c}{ Cultivated land } & \multicolumn{4}{c}{ All } \\
\cline { 2 - 13 } & $r$ & MAE & RMSE & $r$ & MAE & RMSE & $r$ & MAE & RMSE & $r$ & MAE & RMSE \\
\hline CLSM & 0.942 & 0.007 & 0.009 & 0.868 & 0.011 & 0.019 & 0.938 & 0.008 & 0.016 & 0.938 & 0.009 & 0.014 \\
MCD43A1 & 0.938 & 0.010 & 0.010 & 0.812 & 0.014 & 0.022 & 0.911 & 0.012 & 0.019 & 0.914 & 0.012 & 0.016 \\
\hline
\end{tabular}

experimental results demonstrate that the CLSM is robust to the kernel weights estimation. However, errors in the retrieved kernel weights have not been examined in this paper. Additional work is still required to solve several issues. Currently, only one year of the MCD43A1 products was used to obtain a priori knowledge, which may be insufficient. Therefore, more stable and accurate a priori knowledge is required. Besides, we only obtained kernel weights in the blue band. In the future, we will estimate kernel weights for MODIS spectral bands 1 through 7 by this method.

\section{Acknowledgements}

The MODIS and VIIRS series data are available at the NASA's Earth Observing System Data and Information System (https://earthdata.nasa.gov/eosdis) and to be distributed through the LPDAAC (https://pdaac.usgs. gov/). We would like to thank the EOSDIS team for maintaining the network and data archive.

\section{Funding}

This work was funded by the following grants: National Natural Science Foundation of China [41876107], NSFC-Shandong joint fund project [U1706219], Key Deployment Project of Centre for Ocean Mega-Research 
of Science, Chinese Academy of Science [COMS2019J02], Key Research Program of Frontier Science, Chinese Academy of Sciences [ZDBS-LY-7010], National Key Research and Development Program [2016YFA0600102] and the Open Fund of CAS Key Laboratory of Marine Ecology and Environmental Sciences [KLMEES202005], National Key Research and Development Program of China (2016YFA0600102).

\section{ORCID}

Xinpeng Tian (D) http://orcid.org/0000-0002-1020-0706

\section{References}

Ding, A. X., Z. T. Jiao, Y. D. Dong, Y. Qu, X. N. Zhang, C. Xiong, D. D. He, S. Y. Yin, L. Cui, and Y. X. Chang. 2019. "An Assessment of the Performance of Two Snow Kernels in Characterizing Snow Scattering Properties." International Journal of Remote Sensing 40 (16): 6315-6335. doi:10.1080/01431161.2019.1590878.

Gao, F., X. W. Li, A. Strahler, and C. Schaaf. 2000. "Evaluation of the Li transit kernel for BRDF modeling." Remote Sensing Reviews 19 (1-4):205-224. doi:10.1080/02757250009532419

Jiao, Z. T., A. X. Ding, A. Kokhanovsky, C. Schaaf, F. M. Breon, Y. D. Dong, Z. S. Wang, et al. 2019. “Development of a Snow Kernel to Better Model the Anisotropic Reflectance of Pure Snow in a Kernel-driven BRDF Model Framework". Remote Sensing of Environment 221: 198-209. doi:10.1016/j.rse.2018.11.001.

Jiao, Z. T., C. B. Schaaf, Y. D. Dong, M. Roman, M. J. Hill, J. M. Chen, Z. S. Wang, et al. 2016. "A Method for Improving Hotspot Directional Signatures in BRDF Models Used for MODIS". Remote Sensing of Environment 186: 135-151. doi:10.1016/j.rse.2016.08.007.

Knobelspiesse, K. D., B. Cairns, B. Schmid, M. O. Roman, and C. B. Schaaf. 2008. "Surface BRDF Estimation from an Aircraft Compared to MODIS and Ground Estimates at the Southern Great Plains Site." Journal of Geophysical Research-Atmospheres 113: D20. doi:10.1029/2008JD010062.

Li, X. W., and A. H. Strahler. 1992. "Geometric-Optical Bidirectional Reflectance Modeling of the Discrete Crown Vegetation Canopy - Effect of Crown Shape and Mutual Shadowing." IEEE Transactions on Geoscience and Remote Sensing 30 (2): 276-292.

Li, X. W., F. Gao, J. D. Wang, and A. Strahler. 2001. "A Priori Knowledge Accumulation and Its Application to Linear BRDF Model Inversion." Journal of Geophysical Research-Atmospheres 106 (D11): 11925-11935. doi:10.1029/2000JD900639.

Lucht, W., C. B. Schaaf, and A. H. Strahler. 2000. "An Algorithm for the Retrieval of Albedo from Space Using Semiempirical BRDF Models." IEEE Transactions on Geoscience and Remote Sensing 38 (2): 977-998. doi:10.1109/36.841980.

Lucht, W., and J. L. Roujean. 2000. "Considerations in the Parametric Modeling of BRDF and Albedo from Multiangular Satellite Sensor Observations." Remote Sensing Reviews 18 (2-4): 343-379. doi:10.1080/02757250009532395.

Lucht, W., and P. Lewis. 2000. "Theoretical Noise Sensitivity of BRDF and Albedo Retrieval from the EOS-MODIS and MISR Sensors with respect to Angular Sampling." International Journal of Remote Sensing 21 (1): 81-98. doi:10.1080/014311600211000.

Nicodemus, F. E., J. C. Richmond, J. J. Hsia, I. W. Ginsberg, and T. Limperis. 1977. “Geometrical Considerations and Nomenclature for Reflectance." In 52. National Bureau of Standards Monograph.

Noguchi, K., A. Richter, V. Rozanov, A. Rozanov, J. P. Burrows, H. Irie, and K. Kita. 2014. "Effect of Surface BRDF of Various Land Cover Types on Geostationary Observations of Tropospheric NO2." Atmospheric Measurement Techniques 7 (10): 3497-3508. doi:10.5194/amt-7-3497-2014.

Quaife, T., and P. Lewis. 2010. "Temporal Constraints on Linear BRDF Model Parameters." IEEE Transactions on Geoscience and Remote Sensing 48 (5): 2445-2450. doi:10.1109/TGRS.2009.2038901.

Ross, J. 1981. The Radiation Regime and Architecture of Plant Stands. The Hague Dr. W. Junk Publishers doi:10.1007/978-94-009-8647-3 
Strugnell, N. C., and W. Lucht. 2001. "An Algorithm to Infer Continental-scale Albedo from AVHRR Data, Land Cover Class, and Field Observations of Typical BRDFs." Journal of Climate 14 (7): 1360-1376. doi:10.1175/1520-0442(2001)014<1360:AATICS>2.0.CO;2.

Susaki, J., K. Hara, K. Kajiwara, and Y. Honda. 2004. "Robust Estimation of BRDF Model Parameters." Remote Sensing of Environment 89 (1): 63-71. doi:10.1016/j.rse.2003.10.004.

Tang, S., J. M. Chen, Q. Zhu, X. Li, M. Chen, R. Sun, Y. Zhou, F. Deng, and D. Xie. 2007. "LAI Inversion Algorithm Based on Directional Reflectance Kernels." Journal of Environmental Management 85 (3): 638-648. doi:10.1016/j.jenvman.2006.08.018.

Trigg, S. N., D. P. Roy, and S. P. Flasse. 2005. "An in Situ Study of the Effects of Surface Anisotropy on the Remote Sensing of Burned Savannah." International Journal of Remote Sensing 26 (21): 4869-4876. doi:10.1080/01431160500141923.

Vermote, E. F., N. Z. El Saleous, and C. O. Justice. 2002a. "Atmospheric Correction of MODIS Data in the Visible to Middle Infrared: First Results." Remote Sensing of Environment 83 (1-2): 97-111. doi:10.1016/S0034-4257(02)00089-5.

Vermote, E. F., N. Z. El Saleous, and C. O. Justice. 2002b. “Modis Surface Reflectance Product: Algorithm Description and Product Validation." In EGS General Assembly Conference Abstracts, 5072.

Wang, Z. S., C. B. Schaaf, Q. S. Sun, Y. M. Shuai, and M. O. Roman. 2018. "Capturing Rapid Land Surface Dynamics with Collection V006 MODIS BRDF/NBAR/Albedo (MCD43) Products." Remote Sensing of Environment 207: 50-64. doi:10.1016/j.rse.2018.02.001.

Wanner, W., X. Li, and A. H. Strahler. 1995. "On the Derivation of Kernels for Kernel-Driven Models of Bidirectional Reflectance." Journal of Geophysical Research-Atmospheres 100 (D10): 21077-21089.

Zhang, H., Z. T. Jiao, L. Chen, Y. D. Dong, X. N. Zhang, Y. Lian, D. Qian, and T. J. Cui. 2018. “Quantifying the Reflectance Anisotropy Effect on Albedo Retrieval from Remotely Sensed Observations Using Archetypal BRDFs." Remote Sensing 10 (10): 1628-1648.. doi:10.3390/rs10101628. 